XXXIV. Examination of Astringent Substances (continued). By John Stenhouse, Esq., Ph.D.

Read Februa y 21, 1843.

\title{
Black and Green
}

Tea.

REENand black tea are said by Mulder, the chemist who has most recently examined the subject, to be both derived from plants of the same species. The differences observable in them are, as he alleges, chiefly owing to their being collected at different periods of their growth, and to the greater or less degree of heat with which they are subsequently dried; the black teas being strongly heated upon iron plates, while the green teas are exposed to a comparatively moderate temperature. If this statement is correct, it may serve to explain what has been long observed, that an aqueous infusion of black tea, though quite transparent while hot, becomes muddy on cosling, while an infusion of green tea retains its transparency even when quite cold. 'The reason of this difference probably is, that most of the essential oil of black tea is converted, by the partial roasting it has undergone, into a resinous matter, which though soluble in hot is nearly insoluble in cold water, while the essential oil of green tea, on the contrary, remains nearly unchanged, which is probably the cause both of the clearness of its solution and perhaps also of the more powerful effect which green tea is well known to exert on the animal oconomy.

The aqueous infusion of both green and black tea give dull olive-black precipitates with protosulphate of iron, which on standing become leaden black. Infusions of tea also, when evaporated to dryness and distilled, give crystals of theine which collect on the sides and neck of the retort, while the empyreumatic liquor which passes into the receiver gives pretty distinct indications of containing pyrogallic acid."

The Tannin of Tea.- In order to separate the tannin of tea from the other proximate principles of the plant, its aqueous infusion was precipitated with acetate of lead, and the precipitate carefully washed with hot water. Green tea gave a 
bright yellow precipitate, but that of black tea had a brownishyellow colour. The lead salts were decomposed by sulphuretted hydrogen: the solution of the tannin of green tea had only a slight yellow colour, while that of black tea had a much darker colour, but in other respects the properties of both appeared to be the same. The following are the effects upon them of different reagents:- with solution of gelatine they gave white bulky precipitates, and they also gave copions white precipitates with tartar-emetic. Protosulphate of iron throws down bright bluish-black precipitates, nitrate and chloride of iron, olive black, and acetate of iron purple black precipitates. The solution of the tamnin when evaporated to dryness on the water-bath, became of a reddish-brown colour, and was partially decomposed.

When this tannin was subjected to distillation, it invariably yielded a quantity of pyrogallic acid, which sometimes appeared in crystals upon the sides of the retort, but which more frequently remained dissolved in the empyreumatic liquor which passed into the receiver. In this it was easily detected by the usual reagents. It gave a fine reddish-purple colour when dropped on milk of lime, with protosulphate and protonitrate of iron, a fine indigo-blue colour, and with protochloride, a blue resembling ammoniuret of copper. As the quantity of pyrogallic acid obtained was always much less than that which the same quantity of the tannin of either galls or shumac would have yielded, I was led to suspect that it did not arise from the decomposition of the tannin in the tea, but resulted from some gallic acid with which the tannin was mixed. Of the accuracy of this opinion I was speedily convinced by the following experiment:-On treating a strong solution of the tanniu with nearly half its bulk of sulphuric acid added by little and little at a time, a dark brown precipitate fell consisting of the tannin combined with the acid. It was however much more soluble than the corresponding compound of the tannin of galls. It was collected on a cloth filter, strongly compressed, and washed with a little cold water to free it as much as possible from adhering acid. When subjected to distillation it did not afford the slightest trace of pyrogallic acid, showing evidently that the pyrogallic acid I had previously obtained was not derived from the tannin of the tea. When another portion of the precipitated tannin was boiled with tolerably dilute sulphuric acid, it did not yield any gallic acid, but was changed into a dark brown substance, nearly insoluble in cold, and but very little more so in boiling water. It gave a grayish black precipitate with protosulphate of iron, but was not precipitated either by gelatine or tartar- 
emetic. It dissolved however pretty easily both in alcohol and alkalies, forming dark brown solutions. It is evident therefore that though in some of its properties the tannin of tea agrees pretty closely with that of nut-galls, still the products of its decomposition are essentially different.

The tea was next examined for the gallic acid which it evidently contained, and this I was always able to procure by either of the following methods:--The mixture of tannate and gallate of lead obtained by precipitating a decoction of tea by acetate of lead, was decomposed as before by sulphuretted hydrogen and evaporated to dryness. It was then macerated with a very little cold water which removed most of the tannin, but dissolved scarcely any of the gallic acid. The residue was again dried, reduced to powder and mixed with some sand, was repeatedly agitated with æther in a stoppered bottle. The æthereal solution was then poured off, and almost the whole of the æther was recovered by distillation. The residue when left to spontaneous evaporation deposited crystals, which at first had a yellow colour, but which were rendered perfectly white by a second crystallization. The other process was somewhat more tedious, but by it very small quantities indeed of gallic acid can be detected. It consists in putting a number of bits of prepared skin into the mixed solution of tannin and gallic acid already mentioned, and allowing them to remain for nearly a fortnight till the whole of the tannin is absorbed by the skin. The gallic acid is then precipitated by acetate of lead, and the precipitate having been well washed, first with hot water and then with spirits of wine, is to be decomposed by sulphuretted hydrogen.

When evaporated to dryness and treated with æther as before, crystals of gallic acid are readily obtained, which are at first much purer than those got by the former method. I examined several specimens both of black and green tea, and also one of Assam tea, in every instance with similar results. It is evident, therefore, that tea, besides a species of tannin which gives bluish-black precipitates with protosulphate of iron, invariably contains a small but constant quantity of gallic acid, a constituent which has hitherto been overlooked.

Myrobalans.-The name Myrobalans is applied to the fruit of several East Indian trees, the species of which are, I believe, not yet all accurately determined. That which I examined was the yellow kind, the fruit of the Jerminalia Chebula. The ripe fruit has a brownish-yellow colour, is pearshaped, and deeply wrinkled. It consists of a white pentangular nut containing a small white oily kernel, and is covered 
by a mucilaginous and very astringent husk, nearly two lines in thickness. Each of the fruit weighs from 70 to 100 grains, and of this 50 or 60 grains are husk. It is in the husk that the whole of the astringent matter is contained, and it may be easily separated from the nut by slightly pounding of bruising the fruit. The powder of the husk is dark yellow, and its taste is very sharp and astringent. The colour of its aqueous infusion is deep yellow. With protosulphate of iron it gives a deep bluish-black precipitate, which is rather deficient in lustre. The dullness of the colour is owing to the presence of impurities in the husk, for on purifying the astringent matter by precipitating it with acetate of lead, and then decomposing the lead compound with sulphuretted hydrogen, the solution thus obtained gives as fine a colour as can be procured from infusion of galls. With gelatine it gives a very copious, slightly yellow precipitate, the quantity of astringent matter contained in myrobalans being very considerable. With tartar-emetic it also gives a copious brownishyellow precipitate. With protonitrate and protochloride of iron, it gave bluish-black precipitates, which soon changed to olive-black, and with acetate of iron, a fine purple-black precipitate. When the decoction of myrobalans is evaporated to dryness and distilled, it yields abundance of pyrogallic acid; this I found, however, to be derived, not from the decomposition of the tannin it contains, but from a quantity of readyformed gallic acid. Sulphuric acid occasions a very scanty dark brown precipitate in the infusions of myrobalans, if at all dilute, as the combination which this tannin forms with sulphuric acid is pretty soluble. From concentrated solutions, the tannin is readily precipitated as a yellowish-brown tenacious mass. Having been collected on a cloth filter, and freed as much as possible from adhering acid, it was dried and distilled. It yielded no pyrogallic acid, and scarcely any empyreumatic oil; another portion of the same tannin, though boiled in dilute sulphuric acid, was not converted into gallic acid, but changed into a dark insoluble mass.

Gallic acid may be readily obtained from myrobalans by precipitating its decoction with a solution of glue, filtering and evaporating to dryness. On treating the residue with æther, pouring off the solution, recovering the greater portion of the æther by distillation, and leaving the remainder to spontaneous evaporation, crystals of gallic acid were deposited in a few hours. The quantity of gallic acid in myrobalans is pretty considerable.

Besides tannin and gallic acid, myrobalans contains a good deal of mucilage, and a brownish-yellow colouring matter, 
which Dr. Bancroft states was employed in India in his time as a yellow dye. Myrobalans have long been employed by the calico-printers of India instead of galls, and from the large quantity of astringent matter they contain, I think perhaps they might be worth the attention of the tanners and calicoprinters of this country. A decoction of myrobalans makes a very tolerable ink, which however, as we have already stated, is rather deficient in lustre.

Bistort, Polygonum Bistortus. - The root of this plant, which is pretty common in Scotland, has a pale pink colour internally, but when it is exposed to the air for some time it becomes deep yellow. Its aqueous solution is yellowish at first, but on standing it assumes a fine red colour, and the same effect is immediately produced by boiling it with any of the alkalies. With protosulphate of iron it gives a bluish-black precipitate, a good deal resembling that of galls, but having a bluish-purple shade. Gelatine produces a copious brownish precipitate in a solution of bistort, which shows that the quantity of astringent matter it contains is considerable. With tartar-emetic it gives a brownish-white precipitate. When extract of bistort is evaporated to dryness and distilled, it gives distinct indications of pyrogallic acid. The pyrogallic acid however, as in the case of myrobalans, was derived not from the tannin in bistort, but from a quantity of gallic acid with which it was mixed, for on precipitating the tannin by sulphuric acid, and distilling it alone, not a trace of pyrogallic acid was obtained, and when boiled with sulphuric acid it was not converted into gallic acid.

The gallic acid it contains was easily obtained from bistort by precisely the same process as that already described. Its quantity, compared with that of the tannin in the root, was very considerable.

Besides tannin and gallic acid, bistort contains a brownishred colouring matter, and a quantity of mucilage. Bistort may likewise be made to furnish a very tolerable ink, which appears to stand very well. It has a bluish-purple shade, owing to the reddish colouring matter of the root.

The Cashew Nut.-The outer rind of the Cashew nut, the fruit of the Anacardium longifolium, contains a considerable quantity of a species of tannin which gives bluish-black precipitates with the sulphate, nitrate and chloride of iron, and a bluish-purple precipitate with the acetate. It is also readily precipitated by gelatine, but not by tartar-emetic. This tannin is mixed with a small quantity of gallic acid. The shell of the fruit also contains a good deal of a fatty matter, which is solid at ordinary temperatures and crystallizable. It is easily 
saponified when boiled with an alkali, its compound with soda crystallizes in large scales. This fat contains an acrid substance which vesiccates, but it contains no sulphur. When the fat is first expressed from the nut it is but slightly coloured, but by exposure to the air it becomes first brown and then black, and loses much of its acrimony.

Pomegranate Rind.-The rind of the pomegranate contains a considerable quantity of a species of tannin which precipitates gelatine copiously, but gives only a very feeble precipitate with tartar-emetic; with protosulphate, chloride and nitrate of iron, it gives precipitates which are at first deep blue but almost immedately change to very dark olive. With acetate of iron it gives a purple precipitate. Reuss, who has made an analysis of pomegranate rind, states that he found it to contain a little gallic acid. I have been unable to find any, though I have sought it very carefully.

Larch Bark. - The bark of the larch is employed in Scotland to some extent in tanning. The quantity of taunin it contains is considerable, but the leather made with it is of inferior quality. The aqueous solution of the bark is strongly acid to test paper, and has at first a pale yellow colour, which exposure to the air renders brownish-red; it gives a copious fawn-coloured precipitate with gelatine, but none with tartaremetic. With the sulphate, chloride and nitrate of iron, it gives olive-green precipitates. Acetate of iron throws it down of a bluish-purple colour. Sulphuric acid precipitates it of a reddish-yellow colour. When boiled with the acid it dissolves, and the liquid assumes a fine scarlet colour like the infusion of Brazil wood. The altered tannin precipitates on cooling in beautiful red flocks, as it is but little soluble in cold water. It is very soluble in alcohol and alkalies, and its solutions have a rich scarlet colour, which is the most characteristic reaction of this species of tannin. Larch bark also contains a good deal of mucilage and resinous matter. Bircb bark, alder bark, and tormentil root, contain all of them considerable quantities of tannin, which closely resemble that of larch bark. All these species of tannin are readily precipitated by gelatine, but not by tartar-emetic. They give olivegreen precipitates with most of the salts of iron except the acetate, which throws them down of a bluish-purple colour, which on standing changes to a leaden.gray. When boiled with alkalies they immediately assume a fine red colour, but they differ from the tannin of the larch in not being reddened by sulphuric acid. I think it unnecessary to go into more minute details respecting them, as I have been unable to derive from them any determinate or crystalline compounds. I 
shall leave this subject, therefore, for the present with one or two general observations.

The great difficulty of examining the different species of tannin with a view to classifying them, is chiefly owing to their amorphous nature, to the great similarity of their properties, and to the circumstance, that except in the case of nut-galls and shumac, the products of their decomposition are of a very indeterminate character. We think however that there are good grounds for believing that both nut-galls and shumac contain the same species of tannin, for the effects of reagents upon it are exactly the same, and the products of its decomposition, when boiled with either sulphuric or muriatic acid, when destructively distilled, or when left to spontaneous decomposition, are in every instance identical, from whichever of these sources it has been derived. It is remarkable also that in so many instances, in eight cases out of ten which I have examined, the species of tannin which give bluish-black precipitates with protosulphate of iron are accompanied with larger or smaller quantities of gallic acid. In the present state of our knowledge it is impossible to say whether the gallic acid has originally existed in these substances, or has resulted from the decomposition of the tannin they contain. In the case of galls and shumac the latter opinion is probable enough, as we are easily able to effect this change by artificial means, and it also, as is well known, occurs spontaneously. In the case of the other species of tannin, however, we are still unacquainted with any instance of a similar transformation. It is to be hoped that subsequent researches may yet throw light on this very obscure subject. It is also rather singular that in the case of some of those species of tannin which give green precipitates with salts of iron, a somewhat similar circumstance occurs. Thus the tannin of catechu is accompanied by a crystalline acid body, catechine, which also gives green precipitates with salts of iron. I have likewise observed that in the case of infusions of birch bark, alder bark, \&c., when the whole of the tannin they contain had been removed by gelatine, the clear liquid when filtered still contained a substance which precipitated salts of iron olive-green, just as the tannin had done, and which threw down salts of lead as copious dark yellow precipitates. When the lead salts were decomposed by sulphuretted hydrogen, I obtained an amorphous acid substance of a bright yellow colour, which was soluble in water, alcohol and æether, but which did not appear to be crystallizable. 